\title{
Further Studies on the Degradation of Folic Acid in a Growing Culture of Pseudomonas fluorescens UK-1
}

\author{
JUHANI SOINI and KARIN MAJASAARI \\ Department of Biochemistry, University of Turku, SF-20500 Turku 50, Finland
}

\begin{abstract}
Pseudomonas fluorescens UK-1 has been cultivated aerobically in a mineral medium with folic acid as a sole source of carbon and nitrogen. The accumulation of the degradation products of folic acid in the culture medium has been followed during the growth of the organism. Folic acid begins to split to pterine-6-carboxylic acid and PABGA * at the beginning of cultivation and the folic acid, as such, is exhausted before the middle of the logarithmic growth phase. The consumption of PABGA becomes very significant in the early logarithmic phase at the same time as PABA begins to accumulate in the medium. The utilization of PABA is a slow process, for traces of it can be found even after the organism has been growing for 100 hours, i.e. at the end of the growth cycle. Pterine-6-carboxylic acid is first consumed after the logarithmic phase of growth. Carboxypeptidase activity has been estimated during the early logarithmic phase and compared with the results obtained for other pseudomonads.
\end{abstract}

$\mathrm{P}$ seudomonads are able to utilize numerous organic compounds as sole Pource of carbon and nitrogen. Several studies have been made on the splitting of folic acid and related molecules, but these concentrated on the isolation and characterization of the enzymes catalyzing the breakdown of $N$-acetyl-glutamate linkages. ${ }^{1-4}$. The aim of this investigation was to follow the degradation of folic acid during the growth cycle of Pseudomonas fluorescens UK-1, the initiation of which has been described in an earlier paper. ${ }^{5}$ Chromatographic separation has been used for the detection and identification of the degradation products.

\section{METHODS}

Reagents. All chemicals were guaranteed reagents from Sigma Chemical Co. or AnalaR reagents from The British Drug Houses Ltd.

Culture conditions and preparation of cell extract. Ps. fluorescens UK-1 was preserved and cultivated in $5 \mathrm{mM}$ pantothenate medium as described earlier. ${ }^{5}$ The main cultivation

\footnotetext{
* In this publication PABGA $=p$-aminobenzoyl-L-glutamic acid, $\mathrm{PABA}=p$-aminobenzoic acid.
}

Acta Chem. Scand. 27 (1973) No. 10 
was carried out in a medium which was $2.25 \mathrm{mM}$ in folic acid, this providing the only source of carbon and nitrogen. The cells were separated from $100 \mathrm{ml}$ specimens taken during the growth of the organism and treated as before. ${ }^{5}$

Determination of degradation products. Samples were centrifuged and the clear supernatant reduced to $1 \mathrm{ml}$ at $40^{\circ}$ in a rotating vacuum evaporator in order to study the compounds accumulating in the medium during bacterial growth. $100 \mu \mathrm{l}$ of this solution was pipetted onto Whatman No. 3 paper and chromatographed using a $1: 21 \% \mathrm{NH}_{3}$-propanol mixture as solvent. ${ }^{6}$ After drying, the chromatograms were photographed in ultraviolet light with a Desaga MinUVIS lamp at a wavelength of $254 \mathrm{~nm}$. Parallel chromatograms were diazo-stained." Spots from these chromatograms in which PABA and PABGA became visible were cut off and eluted with $3 \mathrm{ml}$ of $\mathrm{H}_{2} \mathrm{O}$. The colour intensity was measured with a Beckman DB spectrophotometer at a wavelenght of $520 \mathrm{~nm}$. Authentic PABA and PABGA were used as standards. Known amounts of these compounds were chromatographed, eluted and measured for preparing the standard curves.

\section{RESULTS AND DISCUSSION}

Figs. 1 and 2 show that, in a growing culture of Ps. fluorescens UK-1, the degradation of folic acid begins almost immediately after the cells have been transferred into the main culture medium. The first degradation products, PABA and PABGA (Fig. 1), and the pterine moiety (Fig. 2), appear in the medium, indicating that the enzymes catalyzing the degradation are situated in the periplasmic space of the cell. PABGA is detectable in the medium

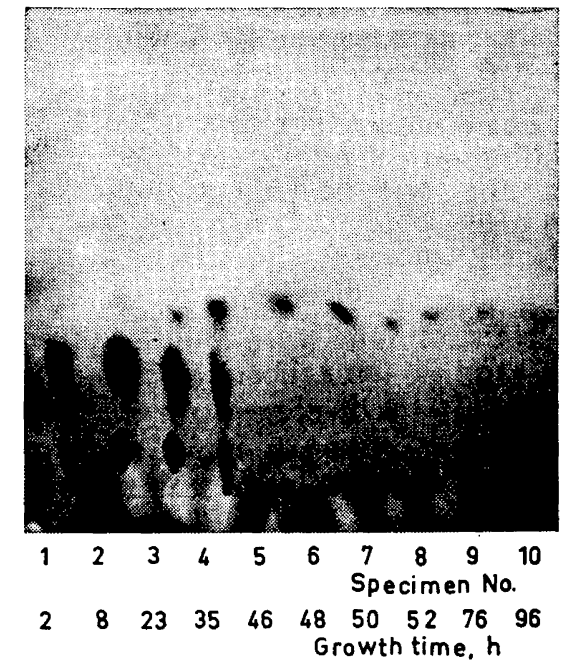

Fig. 1. A diazo-stained chromatogram made from specimens taken from the culture medium during growth of Ps. fluorescens UK-1. Top row, PABA; middle row, PABGA; bottom row, folic acid remaining in the medium.

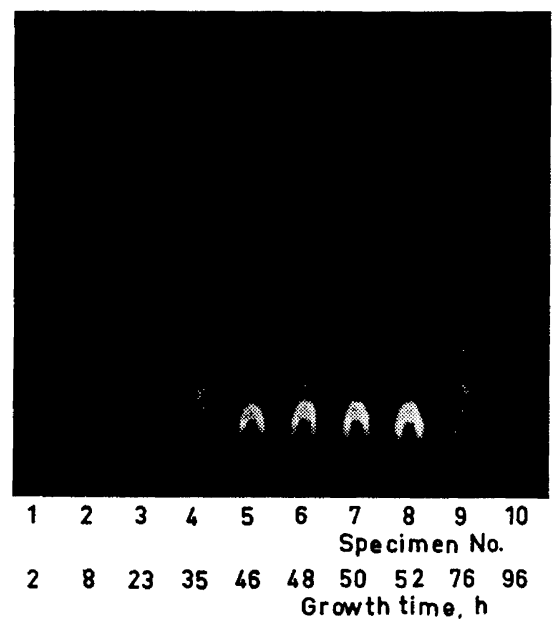

Fig. 2. Same chromatogram as in Fig. 1, but photographed in UV-light before diazo-staining. Dark spots at an $R_{F}$-value of 0.13 in specimens $1-4$ are pterine-6. carboxylic acid almost completely covered by folic acid remaining in the medium. Bright spots at $R_{F}$-value 0.18 in specimens 5-8 are pterine-6-carboxylic acid, which disappears in specimens 9 and 10 .

Acta Chem. Scand. 27 (1973) No. 10 
during the $35 \mathrm{~h}$ after the transfer, and thereafter disappears. PABA starts to accumulate before the PABGA has been exhausted. This is a consequence of the bacterium utilizing the glutamyl residue of PABGA, for traces of PABA remain until the end of the growth period.

The colour intensities of the spots of PABA and PABGA were measured to determine the concentration of these compounds in the culture medium. The results are presented in Fig. 3. After the bond between PABGA and the pterine splits at the beginning of bacterial growth, the utilization of the glutamyl residue of PABGA begins. Thus the amount of PABGA decreases by $15 \mu \mathrm{mol} / 100 \mathrm{ml}$ of growth medium during the first $35 \mathrm{~h}$ while the concentration

Fig. 3. Concentrations of PABGA (curve 1) and PABA (curve 2) in culture medium during the growth of Ps. fluorescens UK-1. Concentrations have been estimated from the spots in the diazo-stained chromatogram (Fig. 1) and calculated as $\mu \mathrm{mol} / 100$ $\mathrm{ml}$ of growth medium.

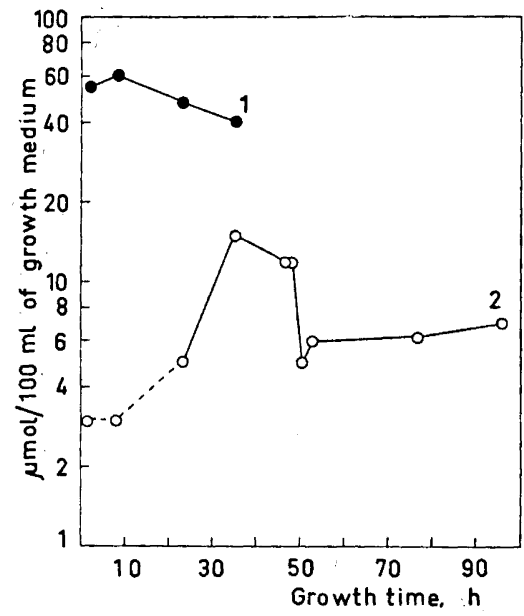

of PABA increases by $12 \mu \mathrm{mol}$. The concentrations of the first two PABA spots have been marked with a dotted line because accurate determination of these faint spots was difficult. After $35 \mathrm{~h}$. Ps. fluorescens UK-I obviously begins to utilize PABA for its concentration in the growth medium diminishes significantly.

The pterine formed from the degradation of folic acid was identified in our earlier paper as pterine-6-carboxylic acid. 5 This compound is present in the growth medium from the beginning of the experiment, but it is mostly covered with intact folic acid in the first four spots of the UV-photograph (Fig. 2). It becomes clearly visible in specimens taken after $35 \mathrm{~h}$, and can be seen in samples over the next $10 \mathrm{~h}$. At the end of the growth period it disappears, clearly indicating that $P$ s. fluorescens UK-1 can utilize pterine-6-carboxylic acid as well as PABGA and PABA. We intend to investigate the utilization of this acid in future studies.

Specimens from the growing culture of $P s$. fluorescens UK-1 were collected between 20 and $30 \mathrm{~h}$ in order to study the velocities with which folic acid is split under in vitro conditions. The chosen time interval was such that PABGA was continuing to decrease and the formation of PABA had clearly started in

Acta Chem. Scand. 27 (1973) No. 10 


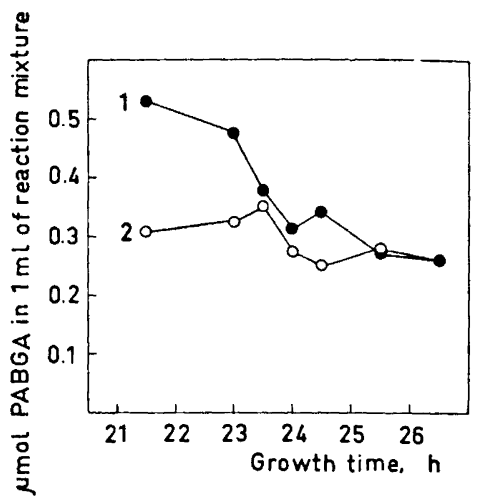

Fig. 4. Formation of PABGA from folic acid after $15 \mathrm{sec}$ (curve 1 ) and $1 \mathrm{~h}$ (curve 2). Enzyme preparations were made from cell specimens taken at intervals during the early logarithmic phase of Ps. fluorescens UK-1 growth. The reaction mixture contained folic acid at $2.3 \mu \mathrm{mol} / \mathrm{ml}$ of $0.05 \mathrm{M}$ TRIS-HCl buffer, pH 7.3. Temperature was $30^{\circ}$ and protein concentration 0.1 $\mathrm{mg} / \mathrm{ml}$. The reaction was started by addition of enzyme and stopped by immersing the tube in an ice bath. $200 \mu$ l of the reaction mixture was pipetted onto Whatman No. 3 paper. After the chromatographic run and diazo-staining, the spots were eluted and the concentrations of PABGA calculated.

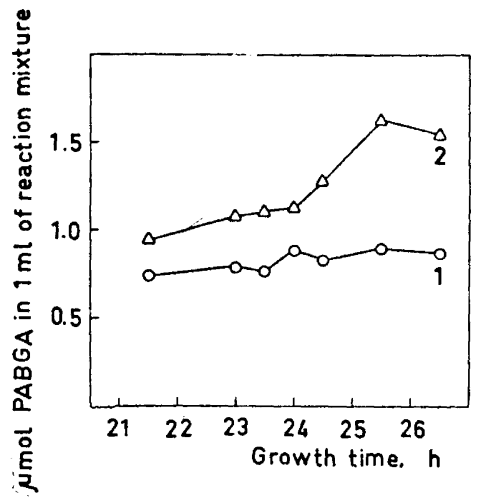

Fig. 5. Formation of PABA from PABGA after $1 \mathrm{~h}$ (curve 1 ) and $3 \mathrm{~h}$ (curve 2). The experimental conditions were the same as in Fig. 4 except that the substrate (PABGA) concentration was $3.8 \mu \mathrm{mol} / \mathrm{ml}$.

the growth medium (Fig. 3). Cells treated with ultrasonic waves and streptomycin were used as enzyme preparation, and folic acid and PABGA as substrates. The results are shown in Figs. 4 and 5. In Fig. 4, curve 1 shows that PABGA is split off from folic acid within 15 seconds after adding the enzyme. The rate of formation of PABGA is probably constant in enzyme preparations made from specimens taken between 21 and $27 \mathrm{~h}$, but the increase of PABA (Fig. 3) during this period leads to an apparent decrease in PABGA concentration. Curve 2 in Fig. 4 shows the PABGA concentration in reaction mixtures after $1 \mathrm{~h}$, and establishes the constant consumption of PABGA.

The splitting of PABGA in cell specimens is presented in Fig. 5 as a function of time. After $3 \mathrm{~h}$ incubation time, the rate of formation of PABA accelerates continually. The maximum value for PABA formation is $1.6 \mu \mathrm{mol} / \mathrm{ml}$ of reaction mixture, indicating that $42 \%$ of PABGA has been converted to PABA. The low value is obviously due to the PABA formed being further metabolized ( $c f$. Fig. 3).

Because of the yellow precipitate produced during the growth of $P s$. fluorescens UK-1, it is difficult to draw any growth curve as a function of time. 
McCullough et al., ${ }^{4}$ however, report a $24 \mathrm{~h}$ generation time for $P$ s. stutzeri when grown on leucovorin, and, according to Goldman and Levy, ${ }^{2}$ a pseudomonad isolated from mud is in the late logarithmic phase after $36 \mathrm{~h}$ when grown on methotrexate. From these it can be estimated that Ps. fluorescens UK- 1 is in an early logarithmic phase between 20 and $30 \mathrm{~h}$. It can be calculated from Fig. 5 that during this period the activity of the enzyme that releases PABA from PABGA increases from a value of 17 milli-units $/ \mathrm{mg}$ to 30 milliunits/mg when ultrasonic and streptomycin treated cells are used. These values are in good agreement with those obtained earlier with a similar enzyme preparation: after protamine treatment, 38 milli-units $/ \mathrm{mg}$ in the logarithmic phase $^{1}$ and 22 milli-units/mg at the end of the logarithmic phase. ${ }^{2}$ In $P s$. stutzeri the value is very high: after $24 \mathrm{~h}$ the activity of carboxypeptidase $\mathrm{G}_{1}$ is 1.8 units $/ \mathrm{mg}$ after protamine treatment. ${ }^{4}$

Acknowledgement. This work has been supported by a grant from the State Committee for the Natural Sciences (Valtion Luonnontieteellinen Toimikunta).

\section{REFERENCES}

1. Levy, C. and Goldman, P. J. Biol. Chem. 242 (1967) 2933.

2. Goldman, P. and Levy, C. Proc. Nat. Acad. Sci. U. S. 58 (1967) 1299.

3. Levy, C. and Goldman, P. J. Biol. Chem. 243 (1968) 3507.

4. McCullough, J., Chabner, B. and Bertino, J. J. Biol. Chem. 246 (1971) 7207.

5. Soini, J. and Majasaari, K. Acta Chem. Scand. 27 (1973) 2115.

6. Goto, M., Forrest, H., Dickerman, L. and Urushibara, T. Arch. Biochem. Biophys. $111 \quad(1965) 8$.

7. Lawson, R., Elliot, W., Elliot, D. and Jones, K., Eds., Data for Biochemical Research, 2nd Ed., Clarendon Press, Oxford 1969, pp. 521-522.

Received July 20, 1973. 